\title{
CURRENT MANAGEMENT OF ENDOMETRIOSIS -AN UPDATE
}

\author{
GANGULY S ${ }^{1}$, BEGUM N $^{2}$, AFROJ S $^{3}$, AKHTER N ${ }^{4}$
}

\begin{abstract}
Endometriosis, defined as the presence of endometrial tissue outside the uterus, is a challenging condition associated with substantial morbidity. Theories on the cause of the disease include retrograde menstruation, coelomic metaplasia, altered immunity, stem cells, and genetics. Management of endometriosis must be individualized according to the desired treatment outcome, whether it is relief of pain, improvement of fertility, or the prevention of recurrence. For alleviation of endometriosis-associated pain, medical treatment is generally successful, with no medical agent being more efficacious than another in spite of significantly differing side-effect profiles. Surgical therapy has also been demonstrated to reduce pain scores in comparison with expectant management, although conservative surgery has been frequently associated with recurrence. Endometriois has been associated with infertility; however, the mechanisms by which it affects fertility are still not fully understood. For treatment of endometriosis-associated infertility, suppressive medical treatment has been proven to be detrimental to fertility and should be discouraged, while surgery is probably efficacious for all stages. Controlled ovarian hyperstimulation with intrauterine insemination is recommended in early-stage and surgically corrected endometriosis. Combined surgery with Gonadotropin-releasing hormone (GnRH) analog treatment has been proposed to be first-line therapy, followed by in vitro fertilization (IVF) as second-line therapy in advanced cases. This article reviews the proposed mechanisms of endometriosis pathogenesis, its current management strategies and its effects on fertility, and treatments of endometriosis-associated infertility.
\end{abstract}

Key words: Endometriosis, Endometriomas, Infertility, Assisted reproductive technologies (ART)

J Dhaka Med Coll. 2014; 23(2) : 245-255.

Endometriosis, a major contributor to pelvic pain and subfertility, is characterized by endometrial-like tissue outside the uterus, primarily on the pelvic peritoneum, ovaries, and rectovaginal septum, and in rare cases on the diaphragm, pleura, and pericardium. Endometriosis affects 6 to $10 \%$ of women of reproductive age, 50 to $60 \%$ of women and teenage girls with pelvic pain, and up to $50 \%$ of women with infertility ${ }^{1-4}$. Endometriosis usually induces a chronic, inflammatory reaction. The condition is predominantly found in women of reproductive age, from all ethnic and social groups. The associated symptoms can impact on general physical, mental and social well-being. Therefore, it is vital to take careful note of the woman's complaints, and to give her time to express her concerns and anxieties as in other chronic diseases. Some women, however, have no symptoms at all ${ }^{4}$.

It is a peritoneal disease, which is dependent on estrogen for growth, derives from retrograde menstruation of steroid hormone"sensitive endometrial cells and tissues, which implant on peritoneal surfaces and elicit an inflammatory response. This response is accompanied by angiogenesis, adhesions, fibrosis,

1. Dr. Shikha Ganguly, Associate Professor, Dhaka Medical College, Dhaka

2. Dr. Nazneen Begum, Assistant Professor, Dhaka Medical College ,Dhaka,

3. Dr. Sultana Afroj, Assistant Professor, Dhaka Medical College ,Dhaka

4. Dr. Nasrin Akhter, Assistant Professor, Dhaka Medical College ,Dhaka

Correspondence: Dr. Shikha Ganguly, FCPS, Associate Professor, Department of Gynaecology and Obstetrics, Dhaka Medical College Hospital, Dhaka, Bangladesh, Mobile- 88-01732605791, e-mail- baren_chakraborty@ yahoo.com 
scarring, neuronal infiltration, and anatomical distortion, resulting in pain and infertility ${ }^{4-6}$. Although most women have retrograde menstruation, not all women with retrograde menstruation have endometriosis; affected women may have an immune dysfunction that interferes with clearing of the lesions. Since

ovarian endometriomas are clonal and lesions can have genetic mutations, somatic mutations with resulting growth dysregulation also may be etiologic factors. Disease at distant sites is probably caused by lymphatic or hematogenous spread or metaplastic transformation ${ }^{4}$.

While underlying cause of endometriosis is uncertain, it is likely to be multifactorial including genetic factors with possible epigenetic influences, perhaps promoted through environmental exposures. It is widely assumed that lesions arise through retrograde endometrial tissue loss during menstruation, coelomic metaplasia and lymphatic spread in immunologically and genetically susceptible individuals. Endometriosis has elements of a pain syndrome with central neurological sensitization and some hallmarks of a neurological disorder ${ }^{7}$, and is a proliferative, estrogen-dependent disorder with growing evidence of progesterone resistance ${ }^{8}$. There is overlap with other conditions characterized by pelvic-abdominal pain and infertility. Some symptomatic women with pelvic pain, who do not have diagnosed endometriosis or who are prior to diagnosis, may benefit from similar treatments. Women with endometriosis typically have a range of pelvic-abdominal pain symptoms, including dysmenorrhoea, dyspareunia, heavy menstrual bleeding, nonmenstrual pelvic pain, pain at ovulation, dyschezia and dysuria, as well as chronic fatigue $^{9,10}$. Endometriosis lesions, particularly deep infiltrating lesions, are often innervated. The presence of endometriotic lesions, followed by denervation and re-innervation, may result in accompanying changes in the central nervous system (central sensitization), creating a chronic pain syndrome ${ }^{7}$. Endometriosis is also associated with infertility, with a strong association between severity of disease and impact on fertility, probably due to impaired tubo-ovarian function, the presence of ovarian endometrioma, subclinical pelvic inflammation, possibly reduced oocyte quality and reduced endometrial receptivity to implantation ${ }^{11}$. Both endometriosis and adenomyosis (lesions occurring in the uterine intramural muscular layer) reduce the chance of success of assisted reproductive treatment ${ }^{12}$. Symptoms of endometriosis contribute substantially to the burden of disease and add substantial cost to society through reduced economic and personal productivity ${ }^{10,13,14}$ While symptoms and examination findings may suggest endometriosis, the gold standard for making the diagnosis remains the laparoscopic visualization of lesions preferably with histologic confirmation ${ }^{10,15,16}$. In the absence of histological sampling, the false-positive rate with laparoscopic visualization alone may approach $50 \%$ especially in women with minimal or mild endometriosis ${ }^{17-19}$. Laparoscopy also enables endometriosis to be staged by the revised American Society for Reproductive Medicine r-ASRM, 1997 scoring system, the 'scoring' system most commonly in current use, objectively defining the disease as minimal (stage I), mild (stage II), moderate (stage III) or severe (stage IV) based on its laparoscopic appearance ${ }^{17-20}$. It is recognized that the stage/extent of disease may not correlate with symptoms experienced, reproductive outcome or recurrence risk ${ }^{20,21}$. Much research has recently focused on serum biomarkers, including cancer antigen-125 (CA125), leptin, monocyte chemotactic protein1 (MCP-1), regulated on activation normal $\mathrm{T}$ cell expressed and secreted (RANTES) and macrophage migration inhibitory factor (MIF), although these have not been useful diagnostic predictors owing to poor sensitivity or specificity, small sample size or inadequate validation of their accuracy. Recent interest has focused on endometrial immunohistochemistry for nerve fibre density and on urinary markers (cytokeratin 19, urinary peptide $1.8 \mathrm{kDa})$. These less invasive diagnostic tests require future formal and robust evaluation of their accuracy ${ }^{20-24}$. 


\section{Risk Factors of Endometrosis}

Risk factors for endometriosis include obstruction of menstrual outflow (e.g., mullerian anomalies, exposure to diethylstilbestrol in utero, prolonged exposure to endogenous estrogen (e.g., because of early menarche, late menopause, or obesity), short menstrual cycles, low birth weight, and exposure to endocrine-disrupting chemicals ${ }^{4,7,9}$. Twin and family studies suggest a genetic component ${ }^{1,4}$. Consumption of red meat and trans fats is associated with an increased risk of laparoscopically confirmed endometriosis, and eating fruits, green vegetables, and n"3 long-chain fatty acids is associated with a decreased risk ${ }^{1,4-7}$ Prolonged lactation and multiple pregnancies are protective. Endometriosis is associated with increased risks of autoimmune diseases and ovarian endometrioid and clear-cell cancers, as well as other cancers, including non-Hodgkin's lymphoma and melanoma ${ }^{4}$. Follow-up of women with pelvic pain and laparoscopically identified disease has shown that 17 to $29 \%$ of lesions resolve spontaneously, 24 to $64 \%$ progress, and 9 to $59 \%$ are stable over a 12 -month period. Endometriosis is a major cause of disability and compromised quality of life in women and teenage girls ${ }^{2,4,6-11}$.

\section{Evaluation of Patient with Endometriosis}

Chronic pelvic pain accounts for $10 \%$ of outpatient gynecologic visits ${ }^{4,25}$. A complete medical, surgical, social, and family history should be obtained from patients who present with this symptom, and they should undergo a physical examination that includes a pelvic examination. Focal pain or tenderness on pelvic examination is associated with pelvic disease in $97 \%$ of patients and

with endometriosis in $66 \%$ of patients ${ }^{4}$. A pelvic mass, immobile pelvic organs, and rectovaginal nodules are suggestive of endometriosis but are not diagnostic because of their poor sensitivity and specificity. An evaluation of both the female patient and her male partner is indicated in cases of associated infertility ${ }^{26}$.

Pelvic pain due to endometriosis is usually chronic (lasting $\geq 6$ months) and is associated with dysmenorrhea (in 50 to $90 \%$ of cases), dyspareunia, deep pelvic pain, and lower abdominal pain with or without back and loin pain. The pain can occur unpredictably and intermittently throughout the menstrual cycle or it can be continuous, and it can be dull, throbbing, or sharp, and exacerbated by physical acitivity ${ }^{25,26}$. Bladder- and bowel-associated symptoms (nausea, distention, and early satiety) are typically cyclic ${ }^{27,28}$. Pain often worsens over time and may change in character; infrequently, women report burning or hypersensitivity, symptoms that are suggestive of a neuropathic component ${ }^{4}$ Symptoms overlap with those of several other gynecologic conditions (e.g., pelvic inflammatory disease, pelvic adhesions, ovarian cysts or masses, leiomyomata, and adenomyosis) and nongynecologic conditions and factors (e.g., irritable bowel syndrome, inflammatory bowel disease, interstitial cystitis, myofascial pain, depression, and a history of sexual abuse), making diagnosis challenging 4-6,27,28.

\section{Different modalities of Treatment of Endometriosis}

Treatment of endometriosis fall into four general categories:

Pain management

Hormone Therapy

Surgical management

Novel strategies

\section{Pain Management}

Pain medications may work well if pain or other symptoms are mild. These medications range from over-the-counter pain relievers to strong prescription pain relievers. The most common types of pain relievers are nonsteroidal antiinflammatory drugs, also called NSAIDS and opioids those interact directly with the nervous system $^{1-3}$.

Evidence on the effectiveness of these medications for relieving endometriosisassociated pain is limited. Understanding which drugs relieve pain associated with endometriosis could also shed light on how endometriosis causes pain ${ }^{7}$. 
Major Guidelines from Professional Societies for the Diagnosis and Management of Endometriosis-Related Pain and Infertility.

\section{Condition Recommendation}

Pain

Diagnosis Surgery is the preferred method for the diagnosis of pelvic pain and a pelvic mass (e.g., endometrioma), but it is not required before initiating empirical therapy, after consideration of other conditions in a differential diagnosis. There should be a low threshold for the evaluation of endometriosis in adolescents because the diagnosis is often missed in this age group.

Treatment Initial treatment is a trial of nonsteroidal antiinflammatory drugs and hormonal therapy (combined oral contraceptives). All hormonal drugs that have been studied (combined oral contraceptives, progestins, GnRH agonists, and danazol) are similarly effective, but their side effects and costs differ. If a GnRH agonist is used, estrogen-progestin add-back therapy is recommended; GnRH agonists are not recommended for adolescents because of their effects on bone. The levonorgestrel intrauterine system is effective in selected patients. Laparoscopic uterosacral nerve ablation is not effective.

\section{Infertility}

Diagnosis Both the male and female partner should undergo a full evaluation.

Treatment Superovulation with intrauterine insemination provides a benefit. Ovarian suppression is not effective in promoting spontaneous pregnancy. The use of a GnRH agonist for 3-6 months before in vitro fertilization and surgical ablation of endometriosis for stage I or II disease are beneficial. Excision of endometriomas $>3 \mathrm{~cm}$ in diameter is of benefit, although there is potential for diminished ovarian reserve.

Adapted from N Engl J Med 2010;362:2389-98

World Endometriosis Society Montpellier Endometriosis Consensus Statements, 2013 (21).

\section{Consensus}

(1) Endometriosis diagnosis and management should be incorporated into the primary health care of women worldwide.

(2) Management, including prevention, should be integrated with other women's healthcare strategies in low-resource settings, and may include education, progestin-based contraceptives, family planning and lactation.

(3) Endometriosis should be considered as a possible diagnosis in adolescents with suggestive symptoms..

(4) Endometriosis should be considered an obstetric risk factor and pregnancies managed accordingly.

(5) The relative risk and absolute risk of ovarian cancer amongst women with endometriosis is so low as not to justify routine ovarian cancer screening.

(6) Well-tolerated, low-cost, easily accessible options such as non-steroidal anti-inflammatory drugs (NSAIDs), other analgesics, combined OCP and progestins should be considered for first-line medical treatment of laparoscopically diagnosed endometriosis .

(7) The combined OCP is an effective medical treatment to minimise the endometrioma recurrence rate after surgical removal of the cyst.

(8) Second-line medical treatments could include gonadotrophin-releasing hormone agonists (GnRH-a, which should be used with add-back HRT, routinely), the levonorgestrel intrauterine system (LNGIUS) and depot progestins .

(9) Danazol and gestrinone should not be used other than for women, established on these treatments in the absence of side effects, for whom other treatments have proven ineffective.

(10) Aromatase inhibitors might be reasonable as a second-line medical treatment, but more research is required. 
(11) There is no evidence of a benefit of pentoxifylline, anti-TNFa (anti tumour necrosis factor alpha) or raloxifene, on the reduction of pain

(12) Anti-angiogenesis agents are at research level only.

(13). Laparoscopic surgical removal of endometriosis is an effective first-line approach for treating pain related to endometriosis.

(14 Although current RCTs have failed to demonstrate benefit of excision over ablation, it is recommended to excise lesions where possible, especially deep endometriotic lesions.

(15) Laparoscopic surgery for endometriosis should always be undertaken in preference to laparotomy, where possible.

(16) The addition of Laparoscopic Uterine Nerve Ablation (LUNA) to laparoscopic removal of endometriosis does not improve pain relief .

(17) Laparoscopic excision (cystectomy) for ovarian endometriomas is preferred where possible to minimise symptom recurrence and endometrioma recurrence.

(18) Highly specialised surgical expertise is required by surgeons, who undertake surgery for deep endometriosis, and it should be undertaken only within centres of expertise.

(19) Laparoscopic surgical removal of endometriosis improves fertility in stage I and II endometriosis.

(20) Laparoscopic excision (cystectomy) where possible for endometriomas is preferred to laparoscopic ablation (drainage and coagulation) to enhance fertility .

(21) The best surgical approach to deep endometriosis in women with infertility is unclear.

(22 Intrauterine insemination (IUI) with COS is effective in improving fertility in minimal and mild endometriosis, but the role of unstimulated IUI is uncertain.

(23) Although IVF may be less effective for endometriosis than for other causes of infertility, it should be considered for use to improve the success rate above expectant management.

(24) There is insufficient evidence of benefit of laparoscopic surgery prior to IUI/COS.

(25) There is no evidence of fertility benefit from medical treatment-ovulation suppression may delay pregnancy and this is not recommended.

(Adapted from Hum Reprod 2013;28(6): 1552-68. doi:10.1093/humrep/det050)

\section{Hormone Treatments}

Because hormones cause endometriosis patches to go through a cycle similar to the menstrual cycle, hormones also can be effective in treating the symptoms of endometriosis. Additionally, our perception of pain may be altered by different hormones. Hormone therapy is used to treat endometriosis -associated pain. Hormones come in the form of a pill, a shot or injection, or a nasal spray. Hormone treatments stop the ovaries from producing hormones, including estrogen, and usually prevent ovulation. This may help slow the growth and local activity of both the endometrium and the endometrial lesions. Treatment also prevents the growth of new areas and scars (adhesions), but it will not make existing adhesions go away ${ }^{29}$.
Combined oral contraceptives can be used cyclically or continuously for endometriosis related pain and are commonly combined with NSAIDs, although they are associated with a 20 to $25 \%$ failure rate $(29,30)$ This approach is first-line therapy in patients without contraindications to the use of combined oral contraceptives. A randomized, controlled trial showed the superiority of combined oral contraceptives over placebo in decreasing baseline pain scores for dysmenorrhea (by 45 to $52 \%$ vs. 14 to $17 \%, \mathrm{P}<0.001$ ) and the volume of ovarian endometriomas (by $48 \%$ vs. $32 \%, \mathrm{P}=$ $0.04)^{30}$. In women with severe dysmenorrhea who have been treated with cyclic combined oral contraceptives, a switch to continuous combined oral contraceptives reduced pain scores by $58 \%$ within 6 months and by $75 \%$ at 2 years $(\mathrm{P}<0.001)^{31}$. Head-to-head randomized, 
nonblinded trials have shown that medroxyprogesterone acetate is as effective in controlling pain as combined oral contraceptives $^{32}$. In addition, in randomized, nonblinded studies, the levonorgestrel intrauterine system (LNG-IUS), which induces endometrial atrophy and associated amenorrhea, diminished endometriosisassociated pain and dysmenorrhea, as compared with regular follow-up with no treatment or treatment with a gonadotropin releasing hormone (GnRH) agonist after conservative surgery ${ }^{32}$.

Health care providers may suggest one of the hormone treatments described below to treat pain from endometriosis:

Oral contraceptives, or birth control pills. These help to make period lighter, more regular, and shorter. Women prescribed contraceptives also report relief from pain ${ }^{33}$. In general, the therapy contains two hormonesestrogen and progestin, a progesterone-like hormone. Women who can't take estrogen because of cardiovascular disease or a high risk of blood clots can use progestin-only pills to reduce menstrual flow ${ }^{33}$. There are some mild side effects from these hormones, such as weight gain, bloating, and bleeding between periods (especially when women first start to take the pills continuously).

Progesterone and progestin, taken as a pill, by injection, or through an intrauterine device (IUD), improve symptoms by reducing a woman's period or stopping it completely. This also prevents pregnancy. As a pill taken daily, these hormones reduce menstrual flow without causing the uterus lining to grow. As soon as a woman stops taking the progestin pill, symptoms may return and pregnancy is possible. An IUD containing progestin, may be effective in reducing endometriosis-associated pain. It reduces the size of lesions and reduces menstrual flow (one third of women no longer get their period after a year of use) ${ }^{34}$. As an injection taken every 3 months, these hormones usually stop menstrual flow. However, one-third of women bleed several times in the first year of injection use. During these times of bleeding, pain may occur.
Additionally, it may take a few months for a period to return after stopping the injections. When menstruation starts again, the ability to get pregnant returns. Women taking these hormones may gain weight, feel depressed, or have irregular vaginal bleeding ${ }^{32-34}$.

\section{Gonadotropin-releasing hormone (GnRH)} agonists stop the production of certain hormones to prevent ovulation, menstruation, and the growth of endometriosis. This treatment sends the body into a "menopausal" state. GnRH agonists come in a nose spray taken daily, as an injection given once a month, or as an injection given every 3 months. Most health care providers recommend staying on GnRH agonists for only about 6 months at a time, with several months between treatments if they are repeated. The risk for cardiac complications and bone loss can rise when taking them longer ${ }^{7}$ After stopping the GnRH agonist, the body comes out of the menopausal state, menstruation begins, and pregnancy is possible ${ }^{35}$. As with all hormonal treatments, endometriosis symptoms return after women stop taking GnRH agonists. These medications also have side effects, including hot flashes, tiredness, problems sleeping, headache, depression, joint and muscle stiffness, bone loss, and vaginal dryness ${ }^{35}$.

Danazol treatment stops the release of hormones that are involved in the menstrual cycle. While taking this drug, women will have a period only now and then or sometimes not at all. Common side effects include oily skin, pimples or acne, weight gain, muscle cramps, tiredness, smaller breasts, and sore breasts. Headaches, dizziness, weakness, hot flashes, or a deepening of the voice may also occur while on this treatment. Danazol's side effects are more severe than those from other hormone treatment options ${ }^{4,7}$. Danazol can harm a developing fetus. Therefore, it is important to prevent pregnancy while on this medication. Hormonal birth control methods are not recommended while on danazol. Instead, health care providers recommend using barrier methods of birth control, such as condoms or a diaphragm ${ }^{4,7,36}$. Researchers are exploring the use of other hormones for treating endometriosis and the pain related to $\mathrm{it}^{36,37}$. 


\section{Surgical Therapy}

Surgical approaches to relieve endometriosisrelated pain can be used as first-line therapy or initiated after failed medical therapies ${ }^{38}$. Surgical procedures include excision, fulguration, or laser ablation of endometriotic implants on the peritoneum, excision or drainage or ablation of endometriomas, resection of rectovaginal nodules, lysis of adhesions, and interruption of nerve pathways ${ }^{18,19,34-39}$. Randomized, controlled trials have shown that at 6 months, laparoscopic ablation of endometriotic implants is $65 \%$ effective in reducing pain, as compared with a $22 \%$ rate of pain reduction associated with diagnostic laparoscopy alone. A small trial comparing laparoscopic ablation with GnRH agonist treatment showed similar pain reduction with the two approaches ${ }^{4}$. Recurrence of pain requiring therapy is common (in 30 to $60 \%$ of patients) within 6 to 12 months after treatment ${ }^{38}$. A combined analysis of data from two randomized trials involving 164 women that compared laparoscopic excision with drainage or ablation of endometriomas larger than $3 \mathrm{~cm}$ in diameter showed that excision resulted in less frequent recurrence of dysmenorrhea, dyspareunia, and pain, as well as reduced rates of further surgery ${ }^{39}$ An alternative strategy for controlling endometriosis- related pain is interruption of nerve pathways but ablation of a segment of the uterosacral ligament has not proved effective. Randomized, controlled trials have shown the superiority of laparoscopic ablation of endometriotic tissue combined with presacral neurectomy (removal of the nerve bundle within the boundaries of the interiliac triangle) over laparoscopic ablation

alone in improving dysmenorrhea and reducing severe midline pain ${ }^{40}$. Case series have shown that hysterectomy with bilateral salpingooophorectomy provided pain relief in 80 to $90 \%$ of women with debilitating symptoms that were refractory to medical or other surgical interventions; pain was reported to recur in $10 \%$ of the women within 1 to 2 years after surgery ${ }^{4,7-11}$. Postoperative hormone replacement should include both estrogen and a progestagen, since estrogen alone may stimulate growth of microscopic disease ${ }^{4}$.

\section{Novel strategies}

Increasing knowledge about the pathogenesis of endometriosis at the cellular and molecular levels may give us the opportunity to use new, specific agents for treatment, including aromatase inhibitors, and new pharmaceutical agents affecting inflammation, oxidative stress, proliferation, angiogenesis and apoptosis. Many of these promising new agents may prevent or inhibit the development of endometriosis. Current medical management of endometriosis is based on combined oral contraceptives, antinflammatory drugs, danazol, gonadotropin-releasing hormone (GnRH) analogues, progestins and steroids receptor modulators have been extensively used in clinical practice ${ }^{32-36}$. Novel agents, that will hopefully improve the therapeutic potential, include aromatase inhibitors, immunomodulators, anti-inflammatory, antiangiogenetic and antiproliferative agents $^{41,42}$.

The use of aromatase inhibitors for medical management of endometriosis is still experimental, two pilot studies examined pain relief after 6 months of daily treatment with an aromatase inhibitor together with highdose norethindrone acetate or an oral contraceptive. Both showed significant (but not complete) resolution of pelvic pain in women with endometriosis who had not responded to first-line treatment. These women were premenopausal, the progestin or combined oral contraceptive was added to the aromatase inhibitor and bone mass density remained stable over the 6 months of the study. Further research is required to determine if aromatase inhibitors will be safe and effective for longterm use in women with endometriosis pain ${ }^{41}$.

Angiogenesis, i.e. the development of new blood vessels from pre-existing ones, represents an integral part in the pathogenesis of endometriosis. During the last decade, an increasing number of studies have therefore focused on the anti-angiogenic treatment of the disease. Numerous compounds of different substance groups have been shown to exert anti-angiogenic effects on endometriotic 
lesions under experimental in vitro and in vivo conditions. These include growth factor inhibitors, endogenous angiogenesis inhibitors, fumagillin analogues, statins, cyclooxygenase-2 inhibitors, phytochemical compounds, immunomodulators, dopamine agonists, peroxisome proliferator-activated receptor agonists, progestins, danazol and gonadotropin-releasing hormone (GnRH) agonists. However, clinical evidence for their efficacy in anti-angiogenic endometriosis therapy is still lacking ${ }^{42}$. Anti-angiogenic compounds hold great promise for the future treatment of endometriosis because they may inhibit the establishment of new endometriotic lesions in early stages of the disease or after surgical treatment. Further experimental studies, controlled clinical trials in particular, are required now to clarify which compounds fulfil these expectations without inducing severe side effects in patients with endometriosis ${ }^{42}$.

\section{Endometriosis and infertility: patho- physiology and management}

Endometriosis and infertility are associated clinically. Medical and surgical treatments for endometriosis have different effects on a woman's chances of conception, either spontaneously or via assisted reproductive technologies (ART). The cause of infertility associated with endometriosis remains elusive, with current findings suggesting a multifactorial mechanism. The respective advantages of surgery, medical treatment, and ART intertwine complexly in women with these disorders ${ }^{43-46}$. This intricate medley mandates a global approach to optimise every option. Indeed, only such a strategy can oppose a situation that still too often prevails, when the main reason for choice of surgery or ART stems from the primary activity of the doctor who is first consulted.

Medical treatments for endometriosis are contraceptive. Data, mostly uncontrolled, indicate that surgery at any stage of endometriosis enhances the chances of natural conception. Criteria for non-removal of endometriomas are: bilateral cysts, history of past surgery, and altered ovarian reserve. Fears that surgery can alter ovarian function that is already compromised sparked a rule of no surgery before ART. Exceptions to this guidance are pain, hydrosalpinges, and very large endometriomas. Medical treatment-eg, 3-6 months of gonadotropin-releasing hormone analogues-improves the outcome of $\mathrm{ART}^{43}$. When age, ovarian reserve, and male and tubal status permit, surgery should be considered immediately so that time is dedicated to attempts to conceive naturally. In other cases, the preference is for administration of gonadotropin-releasing hormone analogues before ART, and no surgery beforehand. The strategy of early surgery, however, seems counterintuitive because of beliefs that milder non-surgical options should be offered first and surgery last (only if initial treatment attempts fail). Weighing up the relative advantages of surgery, medical treatment and ART are the foundations for a global approach to infertility associated with endometriosis ${ }^{43}$.

Endometriosis-associated infertility is poorly treatable with various forms of surgery. Therefore, ARTs, such as controlled ovarian hyperstimulation, intrauterine insemination, or in vitro fertilization, are commonly used for endometriosis-associated infertility. Endometriosis impairs the efficacy of in vitro fertilization. There is no evidence that ovulation suppression before ART would enhance the efficacy of ART. Resection of minimal/mild endometriosis and/or restoration of the normality of pelvic anatomy may enhance the efficacy of ART, but aggressive ovarian surgery should be avoided, because it reduces the ovarian reserves and the likelihood of pregnancy after ART 34,43,4446. The outcome of ART pregnancy is not affected by the history of endometriosis, but the presence of endometrioma may predispose to preterm birth or fetal growth retardation in ART-induced pregnancies. As a whole, infertility due to endometriosis is best treated with various forms of ART, and the earlier clinicians apply these techniques, the more likely pregnancy may ensue $e^{44-46}$.

In most cases, health care providers will recommend laparoscopy to remove or vaporize 
the growths as a way to also improve fertility in women who have mild or minimal endometriosis. Although studies show improved pregnancy rates following this type of surgery, the success rate is not clear. If pregnancy does not occur after laparoscopic treatment, in vitro fertilization (IVF) may be the best option to improve fertility. Taking any other hormonal therapy usually used for endometriosisassociated pain will only suppress ovulation and delay pregnancy. Performing another laparoscopy is not the preferred approach to improving fertility unless symptoms of pain prevent undergoing IVF. Multiple surgeries, especially those that remove cysts from the ovaries, may reduce ovarian function and hamper the success of $\mathrm{IVF}^{34}$.

Endometriosis causes pelvic pain and infertility. Infertility results from effects of endometriosis exerted in the pelvic cavity, in the ovaries and/or on the uterus. Medical treatment effective on pain and at preventing disease recurrence following surgery is of no use for improving the chances of conceiving naturally. Surgery however improves the chances of conceiving in the 12-18 months afterward ${ }^{34-38}$. Endometriosis through extension of the disease to the ovaries may harm ovarian response to controlled ovarian stimulation (COS) needed in ART. Surgery for endometrioma(s) may further reduce ovarian responses to COS in case of endometriosis. Remarkably however, reduced ovarian responses due to endometriosis are not necessarily associated with reduced oocyte quality and ART outcome ${ }^{45}$. Pre-ART treatment with oral contraceptives (OC) improves ART outcome in case of ovarian endometriosis particularly, if endometriomas are present at the time of oocyte retrieval.

\section{Conclusion}

In patients with endometriosis- associated pain, NSAIDs and cyclical combined oral contraceptives are recommended as first-line therapy in the absence of contraindications. If the pain persists, a switch to continuous combined oral contraceptives for 3 to 6 months or a levonorgestrel intrauterine system is warranted. If these approaches are not effective,
GnRH agonist therapy with estrogen" progestin add-back therapy is appropriate. Laparoscopy would be indicated to evaluate and treat persistent pain, a pelvic mass, or both. The patient should be counseled about the association of endometriosis with infertility, but she should also be reassured that she may not have a problem in conceiving and that treatment for endometriosis- associated infertility is often effective. When surgery and medical treatments fail, or natural conception is impossible because of coexisting tubal disease or altered male characteristics, ART is necessary.

\section{References:}

1. Bulun SE. Endometriosis. N Engl J Med 2009; 360:268-79.

2. Berkley KJ, Rapkin AJ, Papka RE. The pains of endometriosis. Science 2005; 308: 1587-9.

3. Tokushige N, Markham R, Russell P et al. Nerve fibres in peritoneal endometriosis. Hum Reprod 2006;21:3001-7.

4. Giudice LC. Endometriosis. N Engl J Med 2010;362:2389-98.

5. Falcone T, Lebovic DI. Clinical management of endometriosis. Obstet Gynecol. 2011 118(3):691705. doi: 10.1097/AOG.0b013e31822adfd.

6. Allen C, Hopewell S, Prentice A. Nonsteroidal anti-inflammatory drugs for pain in women with endometriosis. Cochrane Database Syst Rev 2005;4:CD004753.

7. Stratton P, Berkley KJ. Chronic pelvic pain and endometriosis: translational evidence of the relationship and implications. Hum Reprod Update 2011; 17:327-46

8. Pabona JM, Simmen FA, Nikiforov MA et al. Kruppel -like factor 9 and progesterone receptor coregulation of decidualizing endometrial stromal cells: implications for the pathogenesis of endometriosis. J Clin Endocrinol Metab 2012;97:E376-E392.

9. Kennedy S, Bergqvist A, Chapron C et al. ESHRE Special Interest Group for Endometriosis and Endometrium Guideline Development Group. ESHRE guideline for the diagnosis and treatment of endometriosis. Hum Reprod 2005;20:26982704 .

10. Nnoaham K, Hummelshoj L, Webster P et al. World Endometriosis Research Foundation Global Study of Women's Health Consortium. Impact of endometriosis on quality of life and work productivity: A multi-centre study across 10 countries. Fertil Steril 2011;96:366-73. 
11. Lessey DA. Assessment of endometrial receptivity. Fertil Steril 2011; 96:522-529.

12. Maubon A, Faury A, Kapella $\mathrm{M}$ et al. Uterine junctional zone at magnetic resonance imaging: a predictor of in vitro fertilization implantation failure. J Obstet Gynaecol Res 2010;36:611-18.

13. Simoens S, Hummelshoj L, D'Hooghe T. Endometriosis: cost estimates and methodological perspective. Hum Reprod Update 2007;13:395404.

14. Simoens S, Dunselman G, Dirksen C et al. The burden of endometriosis: costs and quality of life of women with endometriosis and treated in referral centres. Hum Reprod 2012;27:1292-99.

15. Nnoaham KE, Hummelshoj L, Kennedy SH et al. World Endometriosis Research Foundation Women's Health Symptom Survey Consortium. Developing symptom-based predictive models of endometriosis as a clinical screening tool: results from a multicenter study. Fertil Steril 2012; 98: 692-701

16. Jasonni VM. Endometriosis: necessary and sequential therapeutic procedures and guidelines.Urologia. $2010 ; 77$ Suppl 17:12-5.

17. Wykes CB, Clark TJ, Khan KS. Accuracy of laparoscopy in the diagnosis of endometriosis: a systematic quantitative review. Brit $\mathrm{J}$ Obstet Gynaecol 2004;111:1204-12.

18 Yeung P Jr, Sinervo K, Winer W et al. Complete laparoscopic excision of endometriosis in teenagers: is postoperative hormonal suppression necessary? Fertil Steril 2011;95:1909-12.

19. Yu HT, Huang HY, Soong YK et al. Laparoscopic ovarian cystectomy of endometriomas: surgeons' experience may affect ovarian reserve and liveborn rate in infertile patients with in vitro fertilization-intracytoplasmic sperm injection. Eur J Obstet Gynecol Reprod Biol 2010;152:172-75.

20. Adamson GD. Endometriosis classification: an update. Curr Opin Obstet Gynecol 2011;23: 213-20

21. Johnson NP, Hummelshoj L. Consensus on current management of endometriosis. Hum Reprod 2013;28(6): 1552-68. doi:10.1093/ humrep/det050

22. May KE, Conduit-Hulbert SA, Villar J et al. Peripheral biomarkers of endometriosis: a systematic review. Hum Reprod Update 2010; 16:651-74.

23. Bokor A, Kyama CM, Vercruysse L et al. Density of small diameter sensory nerve fibres in endometrium: a semi-invasive diagnostic test for minimal to mild endometriosis. Hum Reprod 2009; 24:3025-32.
24. Al-Jefout M, Dezarnaulds G, Cooper M et al. Diagnosis of endometriosis by detection of nerve fibres in an endometrial biopsy: a double blind study. Hum Reprod 2009;24:3019-24.

25. Diwadkar GB, Falcone T. Surgical management of pain and infertility secondary to endometriosis. Semin Reprod Med. 2011 ;29(2):124-9. doi: 10.1055/s-0031-1272474.

26. ACOG ( American College of Obstetricians and Gynecologists), Practice Bulletin no. 51: Chronic pelvic pain. Obstet Gynecol 2004;103: 589-605.

27. Practice Committee of the American Society for Reproductive Medicine. Endometriosis and infertility. Fertil Steril 2006; 86:Suppl:S156S160.

28. Evans S, Moalem-Taylor G, Tracey DJ. Pain and endometriosis. Pain 2007;132: Suppl 1:S22-S25.

29. Gelbaya TA, Nardo LG. Evidence-based management of endometrioma. Reprod Biomed Online. 2011 ;23(1):15-24. doi: 10.1016/ j.rbmo.2010.11.013.

30. Harada T, Momoeda M, Taketani Y, et al.Lowdose oral contraceptive pill for dysmenorrhea associated with endometriosis: a placebocontrolled, double-blind, randomized trial. Fertil Steril 2008;90:1583-8.

31. Vercellini P, Frontino G, De Giorgi O et al.Continuous use of an oral contraceptive for endometriosis-associated recurrent dysmenorrheal that does not respond to a cyclic pill regimen. Fertil Steril 2003;80:560-3.

32. Abou-Setta AM, Al-Inany HG, Farquhar CM. Levonorgestrel-releasing intrauterine device (LNGIUD) for symptomatic endometriosis following surgery. Cochrane Database Syst Rev 2006;4:CD005072.

33. Practice Committee of the American Society for Reproductive Medicine. Treatment of pelvic pain associated with endometriosis. Fertil Steril ,2008; 90(Suppl. 3), S260-S269

34. Yeung P P, Shwayder J, Pasic R P . Laparoscopic management of endometriosis: Comprehensive review of best evidence. Journal of Minimally Invasive Gynecology, 2009; 16(3), 269-81

35. ACOG ( American College of Obstetricians and Gynecologists). Management of endometriosis (Practice Bulletin No. 114). Obstetrics \& Gynecology 2010;116(1): 223-36.

36. Porpora MG, Pallante D, Ferro A et al. Pain and ovarian endometrioma recurrence after laparoscopic treatment of endometriosis: a longterm prospective study. Fertil Steril. 2010 ;93(3):716-21. doi: 10.1016/j.fertnstert. 2008.10.018. 
37. Hughes E, Brown J, Collins J J et al. . Ovulation suppression for endometriosis. Cochrane Database of Systematic Reviews 2007; (3): Art. No. CD000155.

38. Jacobson TZ, Duffy JM, Barlow D et al. Laparoscopic surgery for pelvic pain associated with endometriosis. Cochrane Database Syst Rev 2009;4:CD001300.

39. Hart RJ, Hickey M, Maouris P et al. Excisional surgery versus ablative surgery for ovarian endometriomata. Cochrane Database Syst Rev 2008;2:CD004992.

40. Proctor ML, Latthe PM, Farquhar CM et al. Surgical interruption of pelvic nerve pathways for primary and secondary dysmenorrhoea. Cochrane Database Syst Rev 2005;4:CD001896.

41. Luisi S, Ciani V, Pinzauti S et al. New perspectives for the treatment of endometriosis. Arch Perinatal Med 2012;18(1), 22-26.

42. Laschke MW, Menger MD. Anti-angiogenic treatment strategies for the therapy of endometriosis. Hum. Reprod. 2012, doi: 10.1093/ humupd/dms026 First published online: June 19, 2012 .

43. de Ziegler D, Borghese B, Chapron C. Endometriosis and infertility: pathophysiology and management. Lancet 2010;376:730-38. doi:10.1016/S0140-6736(10)60490-4.

44. de Ziegler D, Streuli MI, Borghese B et al. Infertility and endometriosis: a need for global management that optimizes the indications for surgery and ART. Minerva Gynecol 2011;63(4): 365-73.

45. Coccia ME, Rizzello F, Mariani G et al. Impact of endometriosis on in vitro fertilization and embryo transfer cycles in young women: a stage-dependent interference. Acta Obstet Gynecol Scand 2011; 90(11):1232-8. doi: $10.1111 / \mathrm{j} .1600-0412.2011$. 01247.x.

46. Dong $X$, Liao $X$, Wang $R$ et al. The impact of endometriosis on IVF/ICSI outcomes. Int J Clin Exp Pathol 2013;15(9):1911-8. 